\title{
Capture of stereopsis and apparent motion by illusory contours
}

\author{
V. S. RAMACHANDRAN \\ University of California, San Diego, La Jolla, California
}

\begin{abstract}
The removal of right-angle sectors from four circular black disks created the impression of a white "subjective" square with its four corners occluding the disks ("illusory contours"). The display in one eye was identical to that in the other except that for one eye a horizontal disparity was introduced between the edges of the cut sectors so that an illusory white square floated out in front of the paper. When a "template" of this stereogram was superimposed on a vertical squarewave grating ( 6 cycles/deg) the illusory square pulled the corresponding lines of grating forward even though the grating was at zero disparity. (Capture was not observed if the template was superimposed on horizontal gratings; the grating remained flush with the background). Analogous effects were observed in apparent motion ("motion capture"). Removal of sectors from disks in an appropriately timed sequence generated apparent motion of an illusory square. When a template of this display was superimposed on a grating, the lines appeared to move vividly with the square even though they were physically stationary. It was concluded that segmentation of the visual scene into surfaces and objects can profoundly influence the early visual processing of stereopsis and apparent motion. However, there are interesting differences between stereo capture and motion capture, which probably reflect differences in natural constraints. The implications of these findings for computational models of vision are discussed.
\end{abstract}

Figure 1 shows a square defined by illusory contours (Brady \& Grimson, 1981; Coren, 1972; Gregory, 1972; Kanizsa, 1976; Rock, 1983; Schumann, 1904). Such contours can be produced by appropriately aligned black disks from which right-angle sectors have been removed. ${ }^{1}$ The brain interprets this figure parsimoniously as an opaque white square with its four corners occluding the four black disks (and not as four sectored disks that have been deceitfully aligned by the experimenter). One has the strange impression of a contour connecting these aligned edges, even though no contour exists physically-hence the name "illusory contours." Whether these contours are physical, physiological, or truly "subjective" is a much debated semantic issue that need not concern us here. Whatever their epistemological implications, illusory contours provide a compelling demonstration of the principle that a great deal of tacit knowledge about the statistics of the natural world must be built into early visual processing. Collinear edges convey an impression of occlusion because through millions of years of trial and error the brain has learned that collinear edges are usually produced by occluding objects.

Why do illusory contours exist, and how do they influence subsequent visual processing? In a world that is so rich in real contours, it is hard to see what evolutionary advantage would accrue from the ability to construct

Thanks are extended to P. Cavanagh, F. H. C. Crick, D. Hubel, and M. Livingstone for stimulating discussions and to E. Ebbesen for facilities. Experiments 1 through 4 were done in collaboration with P. Cavanagh.

The author's mailing address is: Psychology Department, C-009, University of California, San Diego, La Jolla, CA 92093.

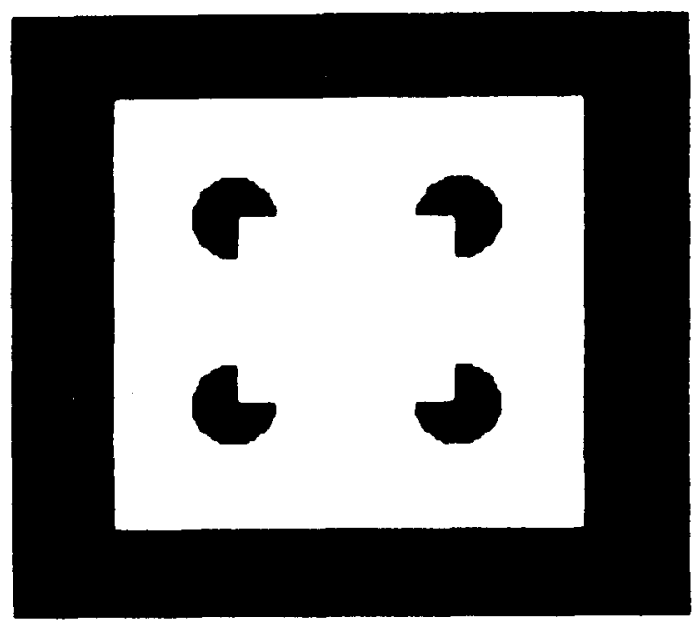

Figure 1. In this figure a square is defined by subjective contours. Observers usually perceive an opaque white square whose corners partially occlude the four black disks. Faint "subjective" lines are seen to delineate the square even though no physical lines exist.

illusory edges. But consider one of our arboreal ancestors' trying to detect a leopard seen against a background of dense foliage. To this creature, segmenting the scene using illusory contours may have been of vital importance as an anticamouflage device. Many animals have developed elaborate splotchy markings in order to break their outlines, and the ability to perceive illusory contours may have evolved specifically to foil this strategy. In fact, it has been argued elsewhere (Ramachandran, 1985b) that many of the early processes of vision (including struc- 
ture from motion, stereopsis, texture discrimination) evolved primarily as tricks to defeat camouflage rather than for seeing depth or motion per se.

It is a well-known fact that the visual system often uses many different strategies for a single purpose. For example, depth perception can be achieved through perspective, accommodation, light and shade, stereopsis (Julesz, 1971), and motion parallax (Rogers \& Graham, 1979), and the organism can make independent use of each of these cues. The existence of multiple strategies suggests that the visual image is normally evanascent and fragmentary, rather than "enriched," although, of course, at any particular instance many cues may indeed coexist fortuitously. If the environment in which we evolved always contained all of these cues, there would have been no incentive (selection pressure) for developing multiple strategies to achieve the same end. We would argue, instead, that it is the intrinsic unreliability of visual information that caused the evolution of parallel mechanisms. At any given instant, the visual system may try to engage all these processes simultaneously to ensure that at least one of them prove reliable. Furthermore, the cues may also interact and complement each other in interesting ways. In this paper we shall explore one specific example of such an interaction, that is, the manner in which illusory contours enhance stereoscopic depth perception. Interactions between illusory contours and motion perception is the subject of a separate communication (Ramachandran, 1985a).

\section{EXPERIMENTS}

\section{Experiment 1: Stereopsis from Illusory Contours}

We constructed a stereogram using two subjective squares, similar to that in Figure 1, by introducing small horizontal disparities between the vertical edges of the cut sectors (Blomfield, 1973; Gregory \& Harris, 1974). The disks themselves were at zero disparity in relation to the surrounding frame. When this pattern (Figure 2) is viewed stereoscopically, the four black disks are seen in the same depth plane as the frame, but one has the distinct impression of a white illusory square standing out clearly in front of the background. On casual inspection, the square looks

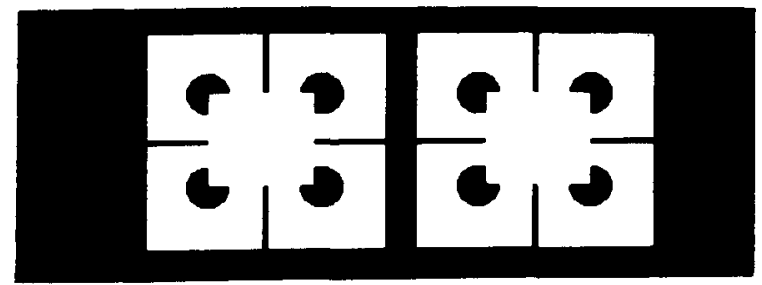

Figure 2. A stereogram is produced by using two subjective squares similar to that of Figure 1. Small horizontal disparities are introduced between the vertical edges of the cut sectors. When the two eyes' patterns are fused, a subjective square stands out in stereoscopic depth. Since most readers find it easier to cross than to diverge their eyes, all stereograms depicted in this paper are printed in reverse, that is, the right eye's picture appears on the left and the left eye's picture on the right. Readers who prefer to diverge their eyes should photocopy and interchange the pictures. opaque rather than transparent; that is, it looks as though it has been cut out from the paper and pushed forward. However, on more prolonged scrutiny it begins to acquire a translucent "glow," and it is difficult to determine whether it looks opaque or transparent.

These stereograms, as well as all subsequent ones described in this paper, were produced using a Macintosh microcomputer. Each eye's picture was a square whose side subtended $5^{\circ}$. The disparity between cut sectors was $20^{\prime}$ of arc, and the width of the illusory squares was $3^{\circ}$. The patterns were printed on white paper and then viewed through a stereoscope by 8 sophisticated observers who were unaware of the purpose of our experiments. They were initially encouraged to view a few sample stereograms for practice and then asked to simply report what they saw in each test stereogram. If they failed to spontaneously report a coherent percept, we prompted them with questions and then recorded their observations. Since we have printed all our stereograms here, readers can directly verify our observations, but, for comparison, we have also summarized in Table 1 the data obtained from our 8 subjects.

\section{Experiment 2: Capture of Stereopsis by Illusory Contours}

Figure 3 depicts a well-known illusion known as the "wallpaper effect." When patterns consisting of repeating stripes are viewed binocularly, they can convey any one of a number of different depth planes (Helmholtz, 1909/1925). However, at any given instant, the entire pattern is seen to occupy only one single plane; the exact plane seen seems to depend mainly on the angle of convergence.

Our basic experimental strategy was to superimpose a "template" of the stereogram depicted in Figure 2 on several kinds of wallpaper to generate "wallpaper stereograms" (Figures 4 through 19). In Figure 4, we simply used repeating vertical rows of spots (see figure captions for additional details). When this pattern was viewed through a stereoscope, the square defined by the subjective contours could be seen standing out clearly in front of the background. Interestingly, the corresponding dot rows on the wallpaper were also carried forward with the plane-an effect we call "stereoscopic capture"' (Ramachandran \& Cavanagh, 1985). This finding implies that the subjective surface in depth created by the subjective contours was somehow pulling the dots with it, even though the dots themselves do not convey any specific disparity information (in fact, they convey zero disparity in relation to the background). All 8 subjects who viewed these patterns reported this effect spontaneously (Table 1).

\section{Experiment 3: Capture of Vertical Stripes}

In Figure 5a, we used continuous vertical lines ( 6 cycles/deg) instead of rows of dots in the background. Subjects reported that the illusion was just as compelling here as in Figure 4. Surprisingly, the capture effect was strong enough to overcome the physical continuity of the 
Table 1

Subjects' Responses to Stereograms

\begin{tabular}{|c|c|c|c|c|c|}
\hline \multirow[b]{2}{*}{ Stereogram } & \multirow[b]{2}{*}{ Percept } & \multicolumn{3}{|c|}{ Number of Subjects } & \multirow[b]{2}{*}{ Comments } \\
\hline & & $\begin{array}{c}\text { Reporting } \\
\text { Spontaneously }\end{array}$ & $\begin{array}{c}\text { Reporting } \\
\text { When Prompted }\end{array}$ & $\begin{array}{l}\text { Not Seeing } \\
\text { the Effect }\end{array}$ & \\
\hline Figure 4 & $\begin{array}{l}\text { Stereo capture (dots pulled } \\
\text { forward) }\end{array}$ & 8 & & & \\
\hline $\begin{array}{l}\text { Figure 5a } \\
\text { (vertical } \\
\text { lines) }\end{array}$ & $\begin{array}{l}\text { Lines pulled forward; illusory } \\
\text { breaks seen on the lines }\end{array}$ & 8 & & & \\
\hline $\begin{array}{l}\text { Figure } 5 b \\
\text { (horizontal } \\
\text { lines) }\end{array}$ & $\begin{array}{l}\text { Lines not pulled forward (remain } \\
\text { flush with the background) }\end{array}$ & 8 & & & $\begin{array}{l}\text { Lines connecting the edges of the cut } \\
\text { sectors themselves were pulled forward } \\
\text { as expected }\end{array}$ \\
\hline Figure 7 & $\begin{array}{l}\text { Portholes with four deeper } \\
\text { corners }\end{array}$ & 4 & 3 & 1 & $\begin{array}{l}\text { Often took a long time }(2-3 \mathrm{~min}) \\
\text { to evolve }\end{array}$ \\
\hline Figure 9 & Capture disappears & 8 & & & \\
\hline Figure 10 & Capture disappears & 8 & & & \\
\hline Figure 11 & Capture disappears & 8 & & & \\
\hline Figure 12 & $\begin{array}{l}\text { The lasso effect; lines pulled } \\
\text { forward but not flush with } \\
\text { illusory square }\end{array}$ & 2 & 6 & & $\begin{array}{l}2 \text { subjects reported that the lines were } \\
\text { pulled forward and were flush with the } \\
\text { illusory square (i.e., percept identical } \\
\text { to that in Figure 5a) }\end{array}$ \\
\hline Figure 13 & Transparency & 4 & 3 & 1 & \\
\hline Figure 14 & Disappearance of transparency & 8 & & & \\
\hline Figure 19 & $\begin{array}{l}\text { Partial capture of rivalrous } \\
\text { elements; (capture less compel- } \\
\text { ling than in Figure 4) }\end{array}$ & 8 & & & $\begin{array}{l}\text { All subjects noted that capture was } \\
\text { less vivid than in Figure } 4\end{array}$ \\
\hline Figure 20 & Capture of lines & 7 & & 1 & $\begin{array}{l}\text { All } 7 \text { subjects noted that capture was } \\
\text { less vivid than in } 5 \text { a }\end{array}$ \\
\hline
\end{tabular}

vertical lines, and caused apparent breaks to appear on the lines at the upper and lower borders of the subjective square, even though all the lines were at zero disparity. (The illusion is especially pronounced with eccentric fixation.) The effect can also be enhanced by using more closely spaced lines (Figure $5 \mathrm{~b}$ ). Here the periodicity of the lines was 12 cycles/deg and the disparity was $10^{\prime}$ of arc.

\section{Experiment 4: Anisotropy of Stereo Capture}

Next we superimposed Figure 2 on horizontal lines (Figure 5c), and found that only the lines actually adjoining the sectors themselves were pulled forward. All the other lines on the square remained flush with the background, and it was very difficult to break the continuity of these lines in order to partition them into two surfaces (Table 1). Perhaps the brain is reluctant to attribute depth to horizontal lines because such lines do not normally convey disparity signals in the real world. The anisotropy was even more striking when we used an illusory diamond instead of an illusory square. The illusory breaks on the lines could now be seen along all four borders of the diamond (Figure 6a) and, as in Figure 5c, the effect disappeared when we used horizontal lines (Figure 6b).

\section{Experiment 5: The Influence of Occlusion Cues in the Perception of Stereo Capture}

The stereograms used in all the experiments described so far convey crossed disparities, that is, the impression of a square standing out in front of the paper (e.g., Figures 3-6). We found that when the disparities were reversed, a curious new percept emerged (e.g., see Figure 7). Sub-

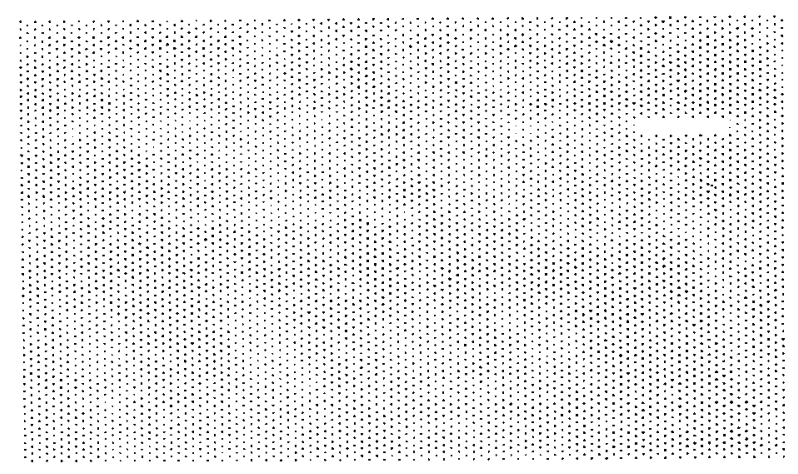

Figure 3. A well-known stereoscopic illusion called the "wallpaper effect" is depicted. If the reader brings the pattern very close to his nose and changes his angle of vergence while viewing this display, he will perceive corresponding changes in the plane of perceived stereoscopic depth. 


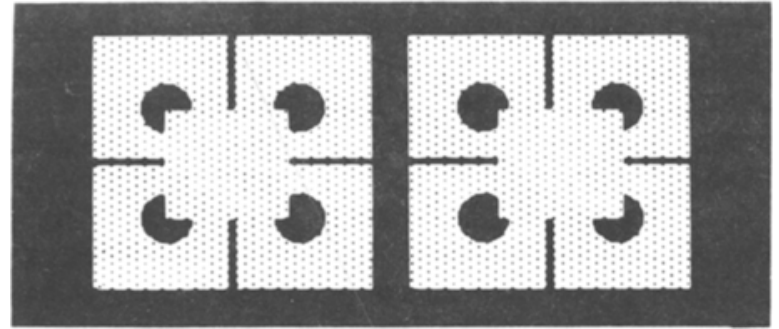

Figure 4. A template of Figure 2 is superimposed on a repeating wallpaper pattern. The interdot separation between the elements constituting the wallpaper is $5^{\prime}$ of arc. A subjective square stands out in front of the background and carries the wallpaper with it even though the elements on the wallpaper are at zero disparity. This is an example of "stereo capture" (see text).

(a)

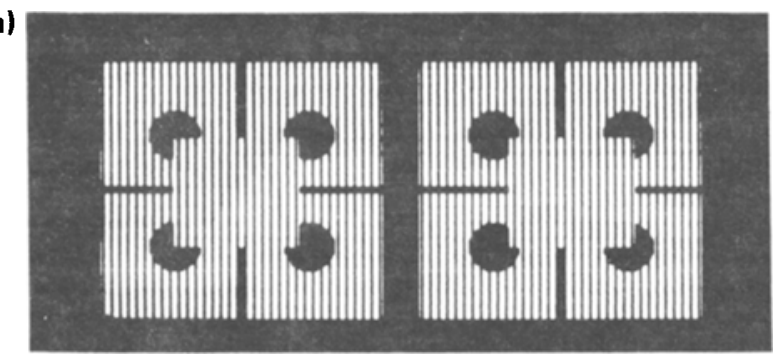

(b)

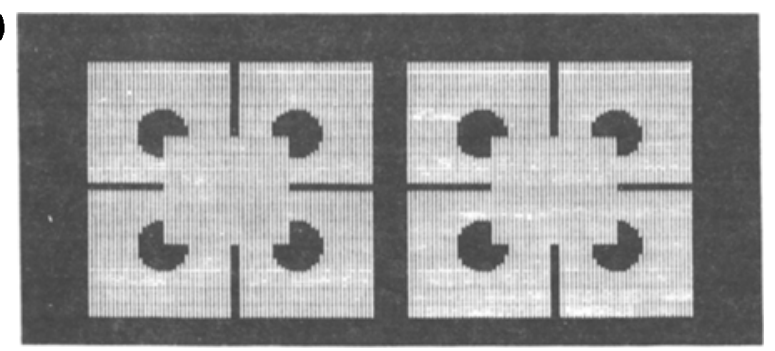

(c)

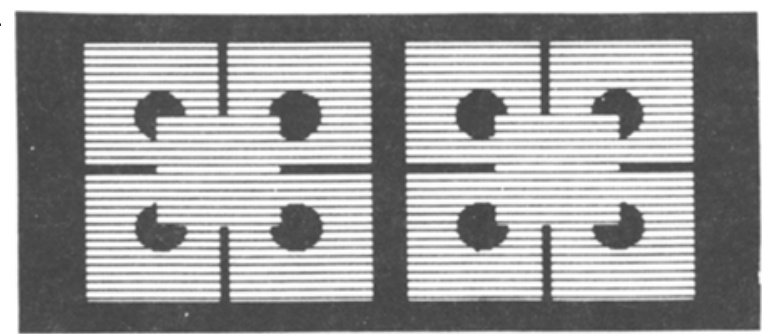

Figure 5. (a) A template of Figure 2 is superimposed on a pattern of repeating vertical stripes. (The spatial frequency of the stripes is 9 cycles/deg.) A subjective square stands out clearly from the background, carrying the lines with it. Also, the capture effect is strong enough to overcome the physical continuity of the lines, and causes apparent breaks to appear on the lines. The effect is especially compelling if the disparity between the cut sectors is arranged to be an exact multiple of the grating periodicity (compare with Figure 12). (b) This figure is similar to (a) except that the disparity has been reduced to $10^{\prime}$ of arc to enable easier fusion. The spatial frequency of the grating is $18 \mathrm{cyles} / \mathrm{deg}$. (c) This figure shows that capture is considerably weaker if horizontal lines are used. The lines adjoining the sectors themselves are pushed forward but the lines in between are not (they remain flush with the background, and it is impossible to see subjective breaks). With eccentric fixation, however, the lines sometimes do appear to come forward. (a)

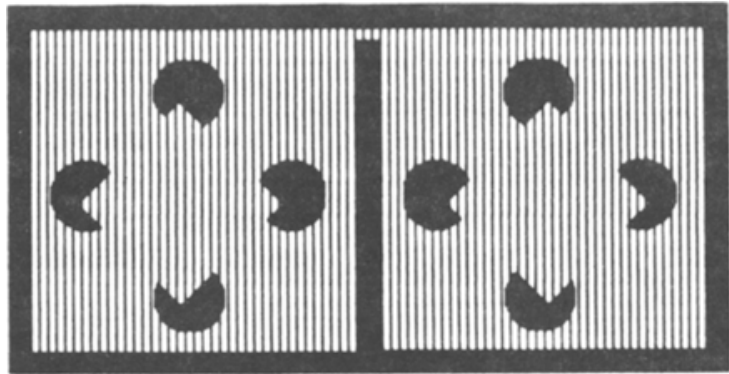

(b)

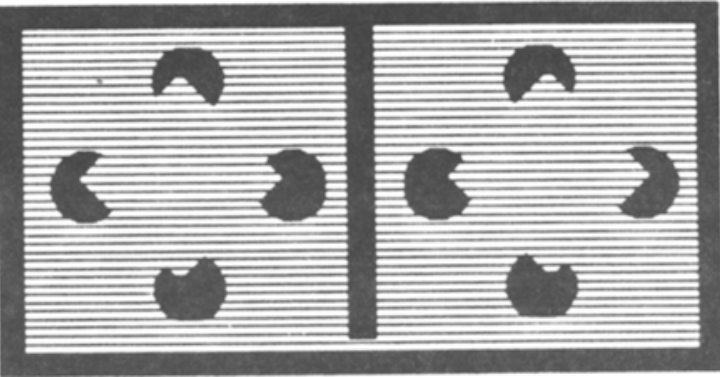

Figure 6. (a) Stereo capture is produced here with illusory diamonds. Apparent breaks can be seen on all four borders of the diamond. (b) This figure shows that horizontal lines are not captured and pulled forward. The lines adjoining the cut sectors themselves are pulled forward, but all the other lines remain flush with the background (Ramachandran \& Cavanagh, 1985).

jects reported that instead of an illusory square they now saw four "portholes" cut out of an opaque sheet of wallpaper. (The black disks which were originally opaque now acquired the subjective quality of being hollow.) Through these holes they could now see the four corners of a smaller square piece of wallpaper, as shown schematically in Figure 8. For many observers, this percept took a long time to "crystallize," but once it was seen, it became quite stable and was perceptually very compelling (Table 1). The illusory contours were now associated with "completion" of the holes (disks) rather than the square, and the corners that were seen through the holes pulled the corresponding lines of wallpaper with them. Note that the illusory contours that complete the portholes are seen only when uncrossed disparities are used, and hence must require the simultaneous use of disparity and occlusion cues. Furthermore, once this illusory surface has been generated, it must in its turn influence the subsequent matching of the finer texture elements in the stereogram.

This unusual percept of seeing four deeper corners is of considerable theoretical interest, for it reinforces the view that it is, indeed, the subjective contours that drive the perceived depth of the wallpaper elements. The implication is that factors such as "occlusion" can directly influence the stereoscopic matching process. It has been known for a long time that occlusion cues can interfere with stereopsis during pseudoscopic viewing of "reallife" stereoscopic photographs. However, occlusion usually vetoes or inhibits a disparity signal during pseudoscopic viewing, whereas what we have here is a very 


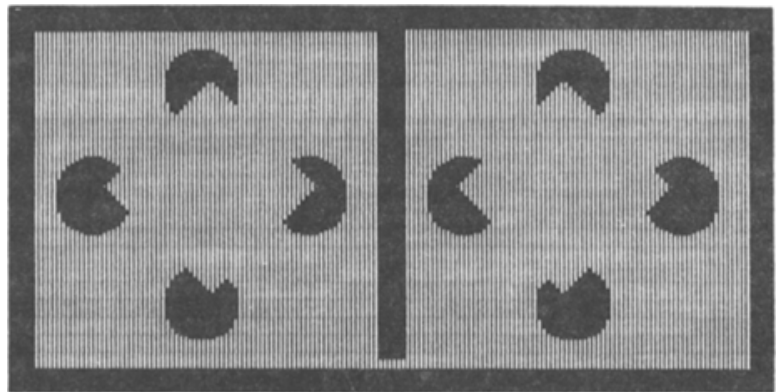

Figure 7. The "porthole" illusion is produced by using uncrossed disparities. Subjects reported seeing four portholes cut out of an opaque sheet of wallpaper. Through these portholes they could see the four corners of a smaller square piece of wallpaper, which pulled the corresponding lines with them (see Figure 8).

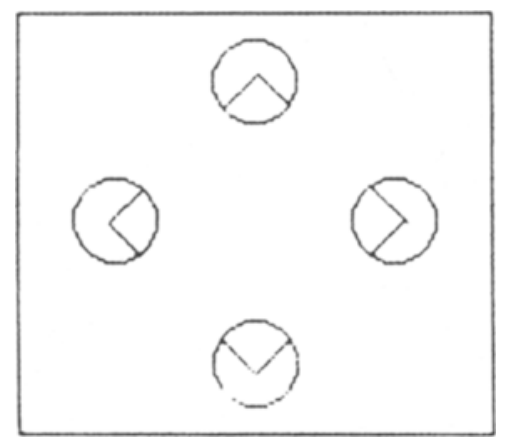

Figure 8. This figure is a schematic view of the percept obtained from Figure 7.

selective enhancement of some disparity signals (and inhibition of others). It is noteworthy that such a striking change in processing disparity signals can be induced by simply interchanging the two eyes' pictures.

\section{Experiment 6: Is the Presence of an Illusory Surface Required for Producing \\ Stereo Capture?}

It is known that unambiguous disparity signals can "propagate". and influence the matching of ambiguous elements in the vicinity (Julesz \& Chang, 1976; Mitchison \& McKee, 1985). This raises the interesting question of whether the stereo-capture illusion was being caused by a similar propagation of disparity signals from the cut sectors or whether the presence of an illusory square is required. Although there is no simple way to answer this question, we tried using "control" stereograms of the kind depicted in Figures 9, 10, and 11. In Figure 9, the cut sectors themselves convey disparities identical to those depicted in our previous stereograms, but they are arranged randomly so that no illusory surface can be perceived. In Figure 10, disparity is introduced between the disks themselves (including the cut sectors) and not just between the cut sectors alone. And lastly, in Figure 11, we have replaced the cut sectors with corners made of little line segments so that no illusory contours are visible.
Stereoscopic capture could not be perceived in any of these stereograms. Some of the lines would occasionally come forward due to convergence, but none of our observers could perceive illusory breaks in the lines. Figure 10 is an especially interesting case, since illusory contours are clearly visible in each eye's picture but are actually destroyed during stereoscopic viewing. The capture illusion disappears completely, even though the disparities conveyed by the cut sectors (and monocular illusory contours) are identical to those used in our previous experiments. This result suggests that the construction of an illusory stereoscopic surface is an important prerequisite for producing stereo capture; the mere propagation of disparity signals will not suffice.

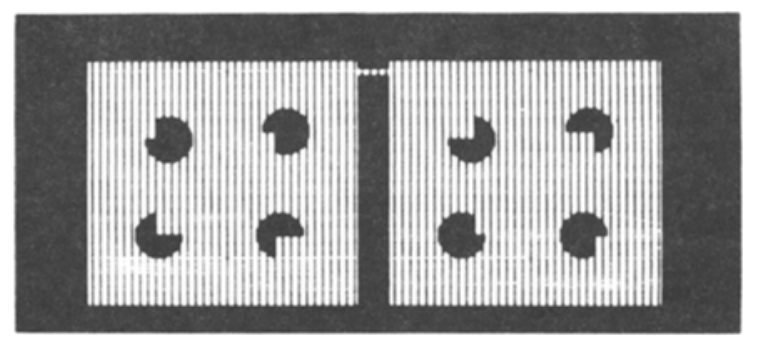

Figure 9. Disparity between the cut sectors is similar to that in Figure $5 \mathrm{a}$, but the sectors are randomly aligned so that no illusory surface is visible in either eye's picture. Stereo capture cannot be obtained.

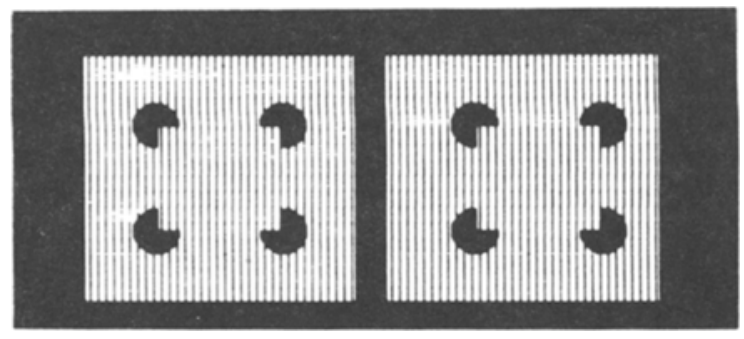

Figure 10. Disparity is introduced between the disks themselves (including cut sectors). Illusory contours are visible in the monocular image but are actually reduced during stereoscopic viewing. Stereo capture is impossible to obtain. Hence, spread of disparity signals from the sectors alone is not sufficient to explain capture; construction of an illusory 3-D surface may be a prerequisite.

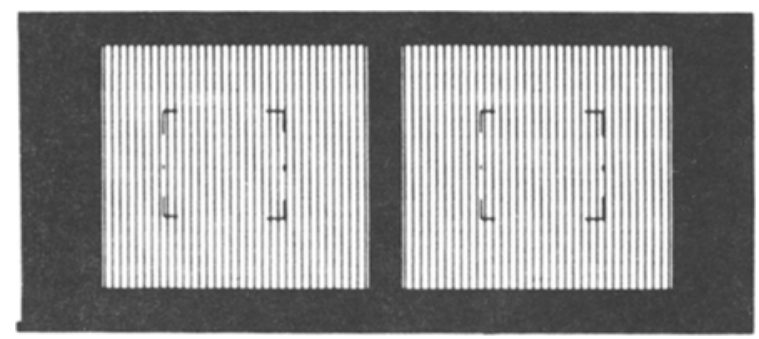

Figure 11. Capture is also abolished if the edges of the cut sectors in Figure $5 a$ are replaced by small vertical line segments. Some of the lines on the wallpaper may occasionally appear to come forward due to convergence, but it is impossible to partition the lines into two surfaces or to perceive illusory breaks in them. 


\section{Experiment 7: Phase Sensitivity of Stereo Capture}

We found that stereoscopic capture was also very sensitive to the spatial phase relationship between the wallpaper and the sectored disks. The cut edges had to be "phase locked" with the lines so that the subjective square's disparity was an exact multiple of the periodicity of the lines. Thus, when the disks themselves were flush with the background, the subjective square occupied exactly the same plane as the enclosed wallpaper that was captured, and this seemed to amplify the illusion. This observation implies that the signal derived from the disparate subjective contours is not merely attributed to the elements of wallpaper; there must be some degree of mutual synergy between the two. In this respect, stereo capture may turn out to be different from motion capture (Ramachandran \& Cavanagh, in press; Ramachandran \& Inada, 1985).

When a phase shift was deliberately introduced so that the disparity of the cut sectors was, say, $2 \frac{1}{2}$ or $3 \frac{1}{2}$ times the periodicity of the lines, a curious "lasso" effect was produced (Figure 12). The lines came forward to occupy a plane corresponding to the nearest integral disparity, for example, 2 or 3 instead of $2 \frac{1 / 2}{2}$ or $31 / 2$. One had the strange impression that the illusory contours were somehow preventing the captured lines from "spilling out" into adjacent regions. However, the effect was somewhat unstable, and the lines had a strong tendency to collapse into the background. In addition, some observers spontaneously reported a "transparency effect"; the illusory square acquired the appearance of a transparent sheet of

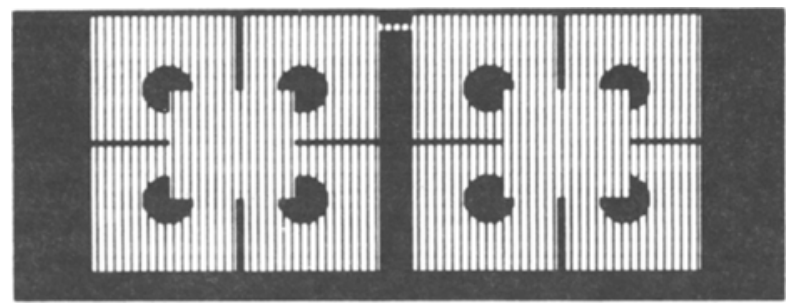

Figure 12. This figure is similar to Figure 5a except that a phase shift was deliberately introduced (see text) to produce the "lasso" effect. Unlike in Figure 5a, however, the disparity between the cut sectors is not an exact multiple of the grating periodicity (disparity $=2 \frac{1}{2} \times$ spacing between lines).

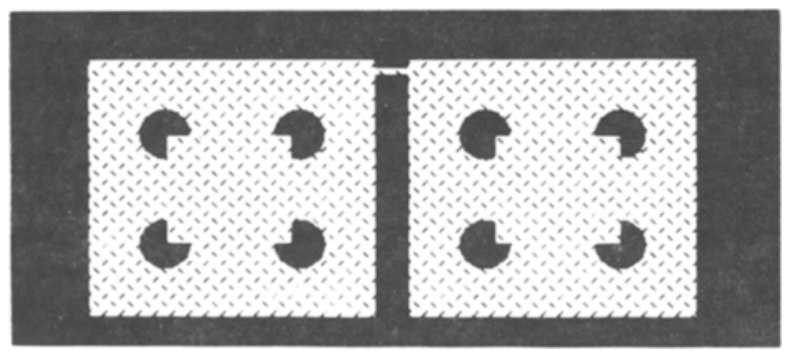

Figure 13. A striking impression of subjective transparency can be produced by combining a two-dimensional texture with an illusory stereoscopic surface that occupies a different plane.

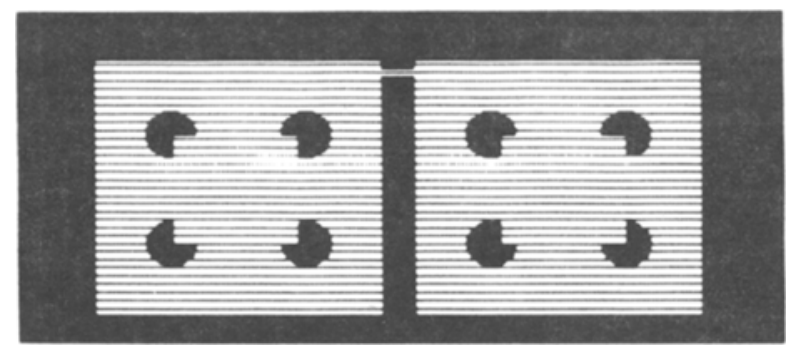

Figure 14. Transparency disappears if horizontal lines are used instead of vertical lines or textures.

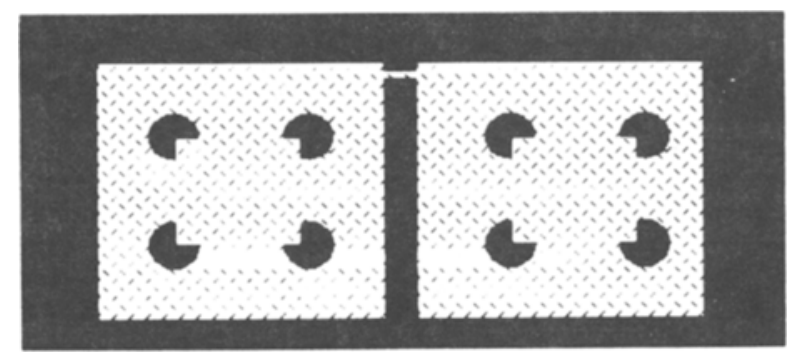

Figure 15. Transparency disappears if disparity is introduced between the disks themselves.

glass lying on a sheet of captured wallpaper. The next three experiments were specifically designed to explore this transparency effect.

\section{Experiment 8: Perception of Transparency in Stereopsis}

The transparency effect reported above can be enhanced considerably by using rich visual textures such as those depicted in Figure 13. Why are such textures especially effective? One explanation might be that the texture is a strong source of disparity signals informing the brain that the wallpaper is at zero disparity. These zero-disparity signals from the texture elements have to be reconciled with the crossed disparity signals arising from the illusory square, and the only logical outcome is the perception of a transparent surface. Consistent with this conjecture, we find the following: (1) The transparency effect is reduced considerably if horizontal lines are used in the background (e.g., compare Figures 13 and 14). This is exactly what one would predict if horizontal lines cannot provide unambiguous zero-disparity signals. (2) Transparency also disappears completely if the two eyes' pictures are interchanged, that is, if one does not perceive a sheet of glass embedded in the background. (3) The effect is abolished if a disparity is introduced between the disks themselves so that they float out from the background (Figure 15). Although this display is logically compatible with a transparency effect (e.g., one might expect to perceive a sheet of glass pulled forward by four sectored disks attached to its corners), subjects never perceive transparency. What is possible logically is not necessarily possible perceptually. 
Experiment 9: Stereo Capture with Opposite Contrast Stereograms

If one begins with a stereogram composed of outline drawings and substitutes one eye's picture with its photonegative, stereopsis can still be perceived. Helmholtz $(1909 / 1925)$ used this demonstration to argue that the stereoscopic system matches contours rather than points of brightness, a conclusion that is also supported by the experiments of Kaufman and Pitblado (1969) and Ramachandran, Rao, and Vidyasagar (1973). We wondered whether the capture effects reported in this paper could also be produced if a contrast reversal was introduced into one eye's picture alone. In Figure 16 we reversed the contrast of all contours in the left eye's picture. This results in rivalry, diplopia, and confusion, and there is no evidence of capture. In Figure 17, on the other hand, we reversed the contrast of the dots alone, and curiously enough this does support capture. Thus, the system will tolerate contrast reversal of the captive elements but not of the cut sectors.

\section{Experiment 10: Can Stereo Capture Overcome Binocular Rivalry?}

It has been known for a long time that stereopsis can coexist with binocular rivalry (Collett, 1985; Kaufman, 1964; Treisman, 1962). In viewing stereograms, such as that shown in Figure 18, through a stereoscope, one perceives the texture boundaries standing out in front, even though the elements defining them are uncorrelated and rivalrous (Ramachandran et al., 1973). Information about the position of the texture boundary can be extracted from a monocular image and used to compute disparity even

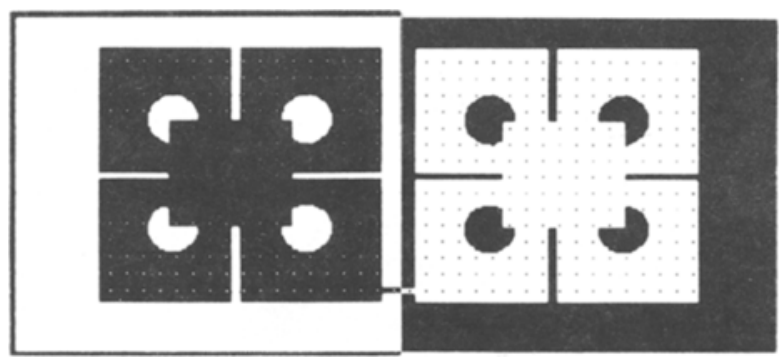

Figure 16. Contrast reversal of one eye's image causes complete disappearance of "capture." This is not true for ordinary Wheatstonetype stereograms (Helmholtz, 1909/1925).

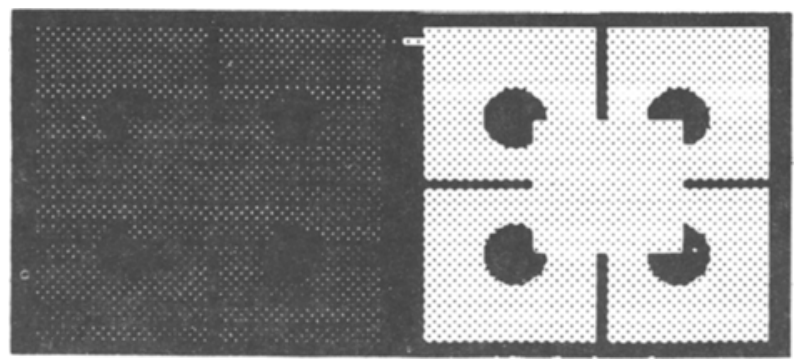

Figure 17. Capture is not abolished if the contrast of the wallpaper alone is reversed without the contrast of the cut sectors' being reversed.
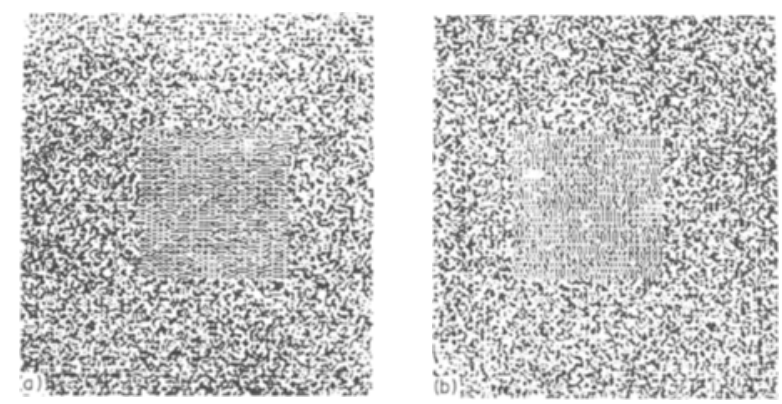

Figure 18. Stereopsis from disparity of visual texture is depicted. The elements defining the textures are completely uncorrelated for the two eyes, but the texture boundary in one eye is shifted horizontally in relation to the other eye. Stereopsis can be obtained in spite of rivalry (Ramachandran et al., 1973), suggesting that some degree of monocular feature analysis must precede stereopsis. The mean luminance of inner and outer textures is identical, that is, they have identical first-order statistics. Readers should diverge their eyes to fuse this stereogram.

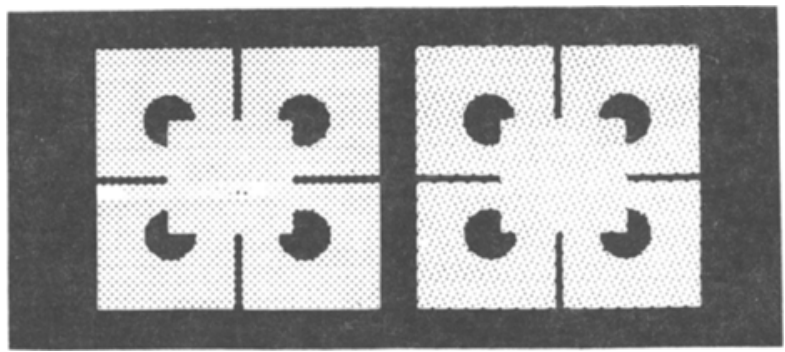

Figure 19. This figure is similar to Figure 5a except that, instead of using vertical stripes, we superimposed two uncorrelated textures on the two images. Stereo capture is much weaker than in Figure 5a. It should be noted that although the textures are dissimilar, they probably have some overlap of their Fourier power spectra. This overlap may produce chance correlations which may explain the partial capture effect seen in these displays.

when that eye's image is being suppressed in consciousness.

These findings raise an interesting question. What would happen if one were to begin with a stereogram such as that shown in Figure 2, and superimpose dissimilar textures on the two eyes' pictures? Figure 19 shows such a stereogram. It can be seen that the illusory edges stand out clearly and many of the texture elements are also pulled forward, but that the capture effect is certainly less compelling than in Figure 4. This is also true if orthogonal gratings are used in the two eyes (Figure 20). Although the lines appeared to come forward, it was difficult to see illusory breaks in them or to partition them into two welldelineated surfaces. These results suggest that although binocular rivalry is not incompatible with stereopsis per se, it does tend to reduce the vividness and clarity of stereo capture.

\section{Experiment 11: The Perception of Complex 3-D Surfaces}

The demonstrations we have described so far have all used a single flat (2-D) plane to generate capture. We find that, by appropriately aligning the cut sectors, it is possi- 


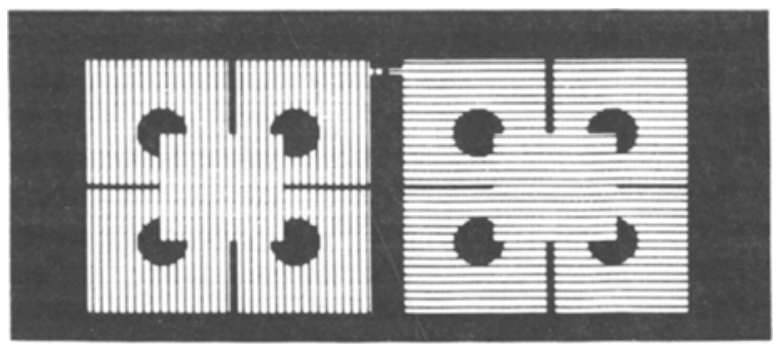

Figure 20. If gratings used in two eyes are orthogonal, stereo capture is reduced but not abolished completely. Interestingly, more capture is seen when the vertical lines are dominant than when the horizontal lines are dominant (during rivalry).

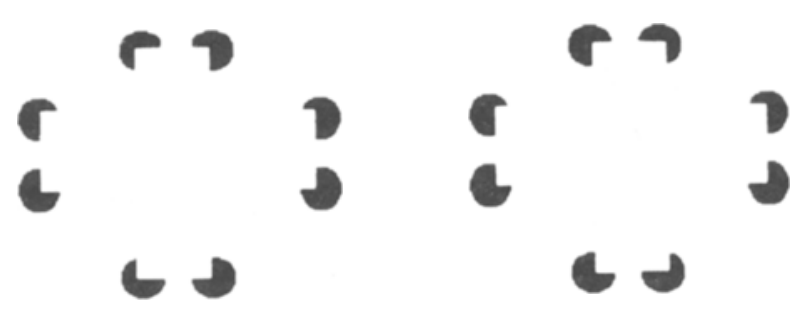

Figure 21. By appropriate alignment of the cut sectors on the disks, it is possible to generate some rather complex illusory 3-D surfaces such as two overlapping bars. The vertical bar appears nearer than the horizontal one.

ble to generate some rather complex surfaces, such as two overlapping bars (Figure 21), curved surfaces, or even two intersecting tilted planes (Figure 22). Would these complex surfaces also capture texture elements from the background? The answer appears to be "yes" for overlapping surfaces but "no" for tilted and curved surfaces, although we have not yet explored this very carefully. When we superimposed the overlapping bars on vertical stripes, capture could be perceived quite clearly (Figure 23). Interestingly, the lines within the overlapping region were always captured by the nearer of the two bars, an observation that is consistent with the occlusion principle discussed earlier (Experiment 5). Again, a simple spread of disparity signals cannot explain this effect, since the region of overlap is equidistant from the cut sectors that define the vertical and horizontal bars. (It should be noted, however, that most of our subjects did not find this "overlap" effect nearly as compelling as the other effects reported in this paper.)

The role of eye movements. We considered the possibility that vergence eye movements were involved in producing these effects. This seems unlikely for two reasons. First, one can obtain a qualitatively different percept by simply reversing the sign of disparity, an outcome that would be impossible to explain on the basis of eye movements alone. Second, we presented the stereograms (Figures 4 and $5 b$ ) in a tachistoscope to 3 subjects. Crossed and uncrossed disparities were flashed in random sequence while the subjects fixated a spot presented on the background just below the subjective square. The duration of each flash was $160 \mathrm{msec}$. When asked to report whether the elements within the square appeared in front of or behind the plane of fixation, they performed the discrimination correctly on 51 of 60 trials ( 3 subjects $\times 20$ trials each). Similar results were obtained with Figure 5 b (54 of 60 responses were correct). In both stereograms, most of the errors in discrimination occurred with uncrossed disparities (7 errors for Figure 4; 4 errors for Figure 5b), which were often incorrectly reported to be in front of the plane of fixation.

These results suggest that eye movements are not responsible for stereo capture. Furthermore, uncrossed disparities are more difficult to process than crossed disparities, perhaps because of conflicting occlusion cues that are inherent in uncrossed stereograms.

\section{DISCUSSION}

Although illusory contours have been studied intensively for many years, there is an unfortunate tendency to relegate them to the status of enigmatic laboratory curiosities or "illusions." Contrary to this popular misconception, we believe them to be of fundamental importance in understanding perception. They provide a striking illustration of the manner in which the brain can achieve image-segmentation using fragmentary evidence-a problem that artificial intelligence (AI) researchers have struggled with for many years.

Our results suggest that, in many instances, the brain may begin by segmenting the scene into contours and surfaces, and that the information derived from such seg-

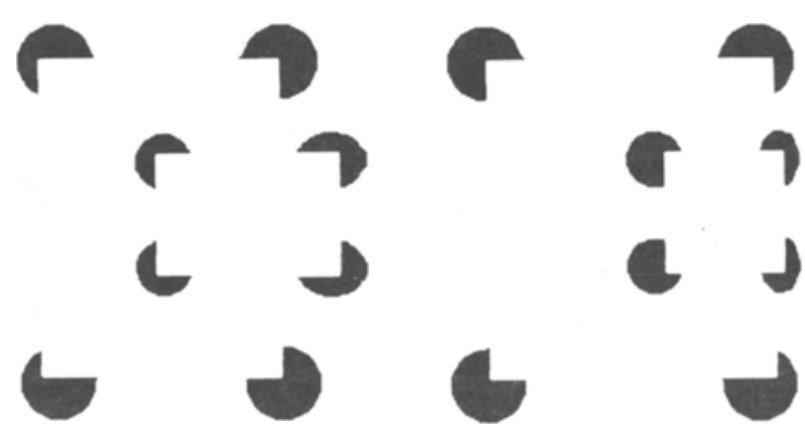

Figure 22. Here a stereogram depicts two tilted intersecting planes.

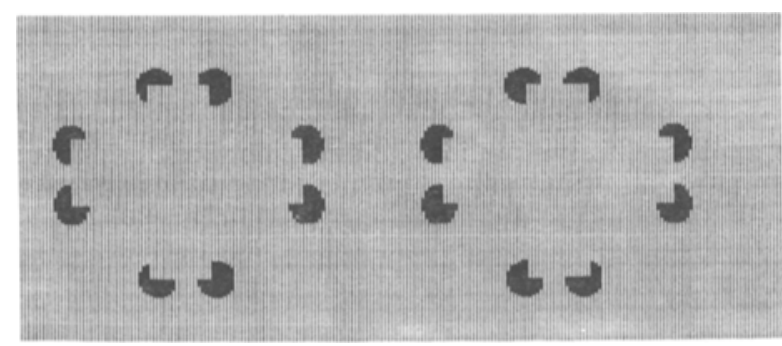

Figure 23. This figure is produced by superimposing a template of Figure 22 on vertical stripes. The lines at the intersection are always captured by the nearer of the two bars-in this case, the vertical bar. This observation suggests that factors such as "occlusion" must influence stereo capture. The effect is especially vivid on eccentric fixation, and tends to fade slightly on direct scrutiny. 
mentation has a profound influence on the processing of retinal disparities. The main findings are: (1) Disparate subjective contours capture regions of zero disparity enclosed within these contours. When vertical stripes are used, the effect is strong enough to overcome the physical continuity of the lines so that illusory breaks appear in them. (2) An anisotropy exists between vertical and horizontal lines, the latter being resistant to capture. On eccentric fixation, however, even horizontal lines are sometimes pulled forward. (3) Capture is also phase sensitive; the effect is most pronounced when the disparity of the illusory contours is an exact multiple of the grating periodicity. (4) Overlay and occlusion can also influence the stereoscopic matching process. A qualitatively different percept consisting of four " portholes" can be produced by simply reversing the sign of disparity. (5) Capture is not seen if a disparity is introduced between the disks themselves rather than between the cut sectors. This observation suggests that capture does not result merely from a spread of disparity signals from unambiguous regions; the creation of a subjective surface is an important prerequisite.

Our stereograms vividly illustrate the manner in which the visual system reconciles information from different depth "cues,"' such as occlusion and disparity, to yield a coherent 3-D percept. Note that the striking changes in perception that we have encountered were all produced by simply introducing very small disparities between the cut sectors. Reversing the sign of disparity (i.e., by simply interchanging the two eyes' pictures) generated a radically different "porthole" percept, whereas introducing a phase shift resulted in a vivid impression of transparency. These observations suggest that the visual system uses a set of simple, but clever, tricks to compute the three-dimensional layout of surfaces. The perception of "transparency," for example, might be signaled only when there is a conjunction of three circumstances: (1) zero disparity signals from the background texture, (2) unambiguous crossed disparity signals from the cut sectors, and (3) inconsistent phase relationship between the texture and the disparate illusory contours. Transparency will not be perceived if any one of these conditions is not fulfilled. For example, if (1) is not true (as when horizontal lines are used, e.g., Figure 14), or if disparities are uncrossed, then transparency is abolished. If (3) is not true, that is, if the disparity of the cut sectors is an exact integral multiple of the grating periodicity, then one observes capture instead of transparency. The detection of a conjunction of (1), (2), and (3) required for transparency need not be "cognitive"; it could be performed just as readily by an AND-gate neuron in one of the extrastriate visual areas.

It is tempting to postulate that capture results from highlevel cognitive processes, in the sense that the signals derived from the illusory surfaces in depth might simply be attributed to the underlying texture elements themselves. This conjecture is refuted by the observation that horizontal lines do not get captured, although their "texture" is just as salient perceptually. The anisotropy and phasesensitivity of stereo capture suggest that the effect cannot arise from mere attribution. We would argue, instead, that there must be some degree of active synergy between signals derived from the illusory contours and those which arise from the wallpaper. Wallpaper composed of vertical stripes (but not horizontal stripes) would excite multiple depth planes simultaneously, whereas the illusory contours would excite only a single depth plane. The latter signal might therefore be used to select, or "highlight," the appropriate plane of wallpaper.

Our conclusions are also consistent with the elegant demonstrations of Mitchison and McKee (1985) and Julesz and Chang (1976), although neither of these studies explored the role of image segmentation produced by illusory contours. Mitchison and McKee found that disparity signals derived from the end-points of two horizontal dot rows viewed binocularly could influence the matching of ambiguous repetitive elements in the middle; that is, there was a spread of disparity signals from unambiguous to ambiguous regions. This "spreading" effect is superficially similar to stereo capture, but there are two important differences: (1) We found that stereoscopic capture could not be produced if disparity was introduced between the disks themselves rather than the cut sectors. This result is important because it implies that a simple spread of disparity signals cannot explain the illusion; the presence of an illusory 3-D surface is an important prerequisite. (2) Under some conditions, Mitchison and McKee observed a dot row that was tilted in stereoscopic space as a result of interpolation-an effect that we could not produce in our displays. When we superimposed a tilted illusory surface on closely spaced gratings (12 cycles/deg), the gratings remained flush with the background and showed no tendency to tilt with the illusory surface.

Differences between stereo capture and motion capture. Our experiments on stereo capture were originally inspired by a very similar set of effects that we have observed in apparent motion ("motion capture"). Two uncorrelated random-dot patterns were visually superimposed and presented in rapid alternation to generate dynamic incoherent noise. When a low-spatial-frequency sine-wave grating was superimposed on the noise patterns and made to jump left and right in synchrony with the alternating dot patterns, all the dots appeared to adhere to the grating and to move with it as a single rigid sheet (Ramachandran \& Cavanagh, in press; Ramachandran \& Inada, 1985). The motion of certain salient image features (such as the grating) masks the incoherent motion signals derived from the spots, and tends to dominate perception by a sort of "winner-take-all" process.

An especially interesting version of motion capture can be produced by a somewhat different procedure which involves the use of "wallpaper," as in stereopsis. We began with illusory squares presented in an appropriately timed sequence to generate apparent motion of an illusory surface (Figure 24; note that the disks themselves 
do not move). When a "template" of this movie was then projected on a regular grid of spots (or stripes), the spots appeared to move with the illusory surface even though they were physically stationary (Figure 25). Since there is no evidence that the dots have not moved, the brain assumes, by default, that they have jumped with the illusory square (Ramachandran, 1985a).

There is a close operational analogy between stereopsis and apparent motion. In both situations, the visual system has to match two slightly dissimilar images that are separated either in space (stereopsis) or in time (motion). Consistent with this analogy we have observed certain similarities between stereo capture and motion capture. For example, our results suggest that the so-called "correspondence problem" in both stereopsis and motion is solved not by cooperative (Marr, 1982) or iterative (Ullman, 1979) algorithms alone, but by using certain salient image features that can be matched unambiguously. However, the manner in which this unambiguous signal is used appears to be somewhat different for the two processes. In motion, the unambiguous signal masks incoherent signals derived from finer image features, and the brain parsimoniously assumes that the latter have also moved with the salient features (or, to put it differently, the unambiguous signal is simply attributed to all features in the image). In stereopsis, on the other hand, the signal derived from salient features must actively facilitate and interact with signals derived from finer image features. This crucial distinction ${ }^{2}$ between stereo capture and motion capture is supported by two pieces of evidence:
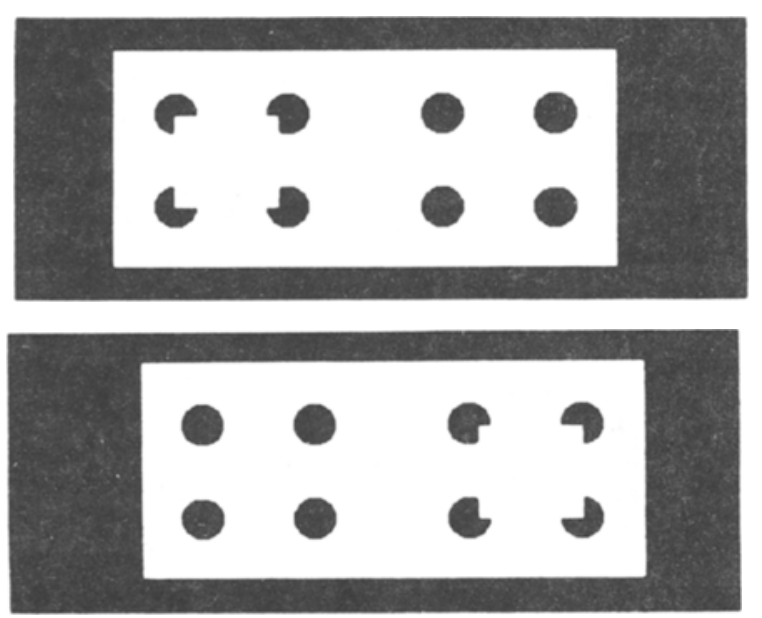

Figure 24. This figure depicts apparent motion of an illusory square (Ramachandran, 1985a). The two frames of the movie are shown one below the other for clarity, but in the original experiment they were optically superimposed so that the disks were in perfect registration. One has the vivid impression of an opaque illusory surface that jumps left and right while covering (and uncovering) the black disks in the background. Note that all eight disks are present in each frame so that the disks themselves do not move. This illusion requires the presence of contours defined by gradients of brightness. If one uses red disks on a green background, at equiluminance, the motion of the illusory square disappears completely and is replaced by the appearance of the disks' simply opening and closing their "mouths" ("Pacmen").
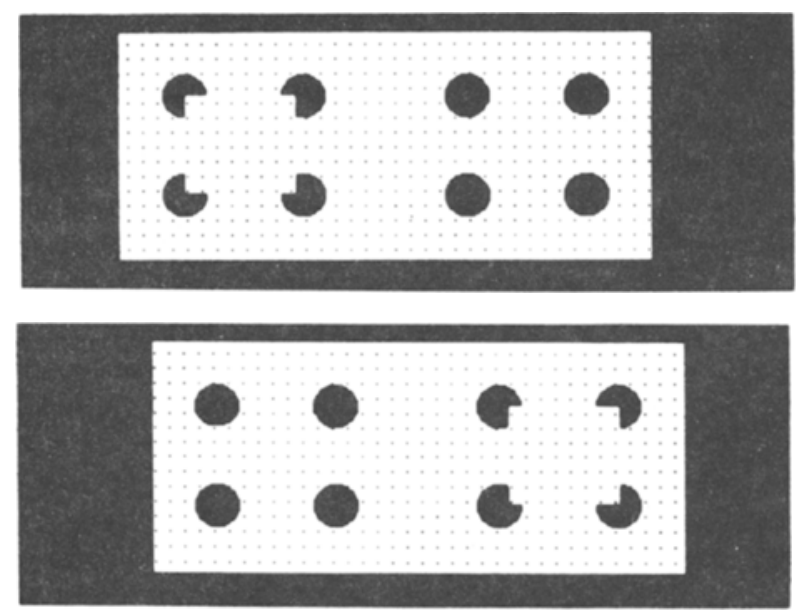

Figure 25. A "template" of the movie described above is superimposed on vertical rows of dots. The dots are present in both frames and are exactly superimposed on each other so that they can generate no movement signals of their own. However, when the illusory square jumps, all the dots corresponding to its surface appear to jump with it as a single solid sheet -an example of a class of illusions that we call motion capture (Ramachandran, 1985a; Ramachandran \& Inada, 1985). The motion of the illusory surface spontaneously generalizes to the dots contained within its boundaries. The reader can view this illusion by using computer software (Apple floppy disk), provided with the recent special issue of the journal Perception (London) (1985; Vol. 14) on human motion perception. The disk is included inside the back cover of that journal.

1. A jumping illusory square will capture horizontal gratings almost as effectively as vertical gratings (Ramachandran, 1985a), and the spatial phase relationships do not have to be accurately maintained across successive positions. This suggests that the signal derived from the illusory surface is simply attributed to the enclosed texture elements. These findings stand in sharp contrast to stereo capture, which, as we noted earlier, is anisotropic and phase-sensitive, observations which imply synergy rather than mere attribution.

2. Even textures that are completely uncorrelated in successive frames can be captured very readily, especially if sine-wave gratings are used (Ramachandran \& Cavanagh, in press; Ramachandran \& Inada, 1985). On the other hand, if the two eyes' pictures are uncorrelated, stereo capture is reduced (Figure 19). The visual system is reluctant to "attribute" the signal derived from the illusory square to the rivalrous texture elements. Unlike wallpaper patterns, uncorrelated textures generate very few disparity signals, and hence there is very little scope for synergistic interaction.

Why is there such a striking difference between the two processes? The logic of the scheme becomes clear if one considers the constraints underlying stereo and motion and the perceptual problems that the two systems were confronted with as they evolved. Consider successive views of a leopard jumping from branch to branch on the treetops while pursuing one of our arboreal ancestors. For long jumps of the leopard, the excursion of the dots is beyond the displacement limit of coherent motion (Braddick, 
1974), giving rise to the question: How does the visual system know which spot goes with which? Our results suggest the following strategy: (1) The motion of certain salient features (e.g., the leopard's outline) masks or inhibits the incoherent signals from the spots, and (2) the signal from the salient features is then spontaneously attributed to the spots as well. This strategy allows the visual system to preserve continuity of object identity while at the same time eliminating spurious motion signals through masking. The only disadvantage is that one could no longer see small local excursions of the spots themselves. For example, any slight ripple of the muscles on the leopard's flanks (or a change in its facial expression) would no longer be detected, but this is a small price to pay if one is trying to run away from it!

Motion capture "works" only because the brain can take advantage of certain statistical properties, such as the fact that moving bodies normally carry their surface textures with them (e.g., spots do not usually fly off leopards). However, contrary to the views of AI researchers, we would argue that the visual system does not make elaborate or sophisticated use of these properties. Instead, the system appears to use a simple set of special-purpose tricks (e.g., capture) to solve the problem (Ramachandran, 1985b). By using appropriately weighted mutual inhibition between motion signals derived from different channels, the visual system solves correspondence and achieves continuity of object identity without benefit of either computation or cognition.

It follows from these considerations that the motion system must, of necessity, have developed a high tolerance for changes in detailed texture. The stereo system, on the other hand, operates under much narrower constraints. First, since the distance between the eyes is constant (and small), the range of binocular disparities encountered is much smaller than the range of transformations encountered during motion of a textured object. Second, since the eyes are separated horizontally, retinal disparities are always horizontal, whereas successive motion transformation can occur along any axis. These narrower constraints may have allowed the stereo system to evolve degrees of refinement that would not be feasible in the motion domain. Since the leopard is not moving and the two eyes are a fixed distance apart, the system can afford the luxury of carefully computing detailed depth relationships.

Possible physiological correlates: Interaction with color and motion. Illusory contours are often thought to be "cognitive"' in origin, but two observations contradict this view. (1) Von der Heydt, Peterhans, and Baumgartner (1984) have recently shown that certain cells in V2 (area 18) of the monkey respond quite strongly to illusory contours, and (2) illusory contours cannot be produced in the absence of luminance contrast between the disks and background texture. For example, if isoluminant color contours (Gregory, 1977), cyclopean targets (Prazdny, 1985), or texture boundaries (Cavanagh, personal communication, 1985) are used to define the cut sectors, then no illusory contours are seen. Also, as noted by Cavanagh, stimuli such as Figure $26 \mathrm{~b}$ do not yield illusory contours, even though they are not logically incompatible with a cognitive "occlusion" hypothesis. (Compare Figures 26a and 26b.)

An important correlation with physiology concerns the separation of color, "form," motion, and stereo channels in early visual processing. When tangential sections of area 18 in the rhesus macaque are stained for cytochrome oxidase, one observes distinct dark stripes corresponding to regions rich in this enzyme. There are two kinds of dark stripes, broad and narrow, which alternate with unstained "interstripe" regions (DeYoe \& Van Essen, 1985; Hubel \& Livingstone, 1985; Shipp \& Zeki, 1985). Single-unit recordings have revealed that cells in the narrow stripes have a double-opponent organization appropriate for detecting color contrast, cells in the broad stripes are tuned to binocular disparity, and

(a)

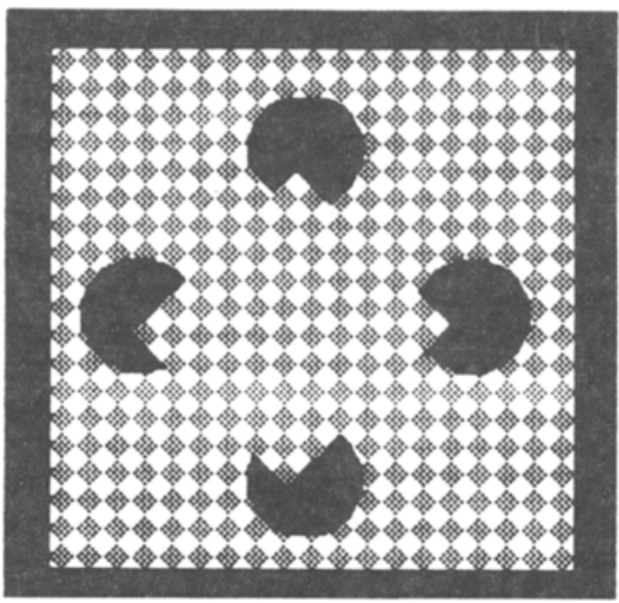

(b)

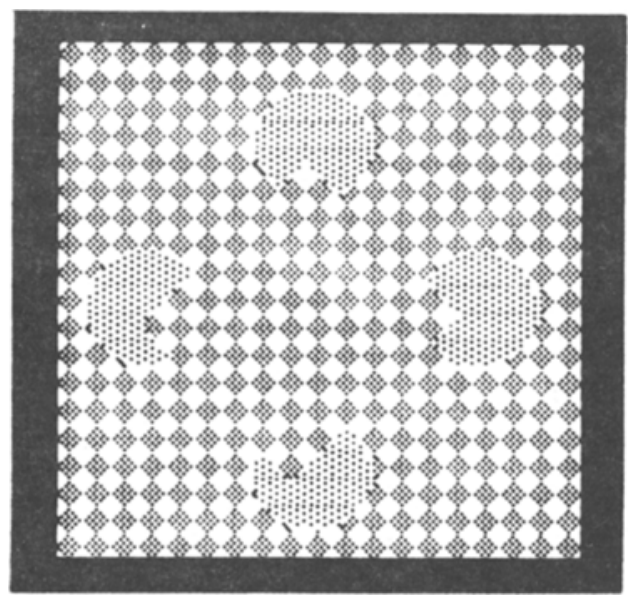

Figure 26. (a) Illusory contours are enhanced by wallpaper. If an illusory square is superimposed on wallpaper, one has the vivid impression of a square patch of wallpaper occluding four disks in the background. The elements of background texture appear to actually enhance the illusory contours. (b) The illusory contours disappear completely at isoluminance, that is, if the luminance of the disks is made identical to the mean luminance of the background texture. An effect similar to this was recently discovered by Cavanagh (1985). 
those in the interstripes are sensitive primarily to bar length ("form"). Cells sensitive to direction of movement, on the other hand, tend to dominate the "magnocellular" pathway that relays through layer 4B in area 17 and terminates eventually in the broad bands in area 18 as well as in area MT. In MT, the cells are tuned to both binocular disparity and motion but are insensitive to color contrast (Van Essen, 1985).

Are there any psychophysical correlates of this anatomical segregation? Lu and Fender (1972) replaced the blackwhite elements of a Julesz random-dot stereogram with red-green elements and found that stereopsis disappeared completely at isoluminance, even though the invidual elements could still be resolved quite clearly. Similarly, in a random-dot kinematogram, if the black-white dots are replaced by colored dots at isoluminance, the appearance of an oscillating central square is either lost (Ramachandran \& Gregory, 1978) or reduced considerably (Cavanagh, Boeglin, \& Favreau, 1985). These results suggest that color boundaries provide only a weak input to stereopsis and motion.

We wondered whether the capture effects described here would also be reduced at isoluminance, and our preliminary results suggest that this is indeed the case. Hubel and Livingstone (personal communication, 1985) have recently observed a reduction in perceived depth when viewing an isoluminant version of the stereogram depicted in Figure 2. Prompted by this observation, we replaced all the black-white contours in Figure 5a with red-green borders at equiluminance. The stimuli were produced on a color monitor using an Amiga microcomputer, and exact equiluminance was achieved by viewing the display through a color-flow filter combined with a rotating polarizer. There was a dramatic perceptual change at equiluminance; stereo capture disappeared completely, and the lines collapsed to the background, although one could still clearly see that the edges of the cut sectors were bent forward. An even more striking effect was observed using an isoluminant version of the kinematogram depicted in Figure 25. When brightness contrast was present, one always saw an illusory square jumping back and forth and carrying the dots with it. At isoluminance, however, this effect disappeared. There was an abrupt transition to seeing the disks ("Pacmen") simply opening and closing their "mouths" and the spots no longer appeared to jump. Taken together, these results suggest that the creation of illusory surfaces associated with motion and stereopsis requires brightness contrast; color contrast will not suffice. Perhaps this dramatic reduction of depth and motion at equiluminance is a direct perceptual consequence of the early anatomical segregation observed by physiologists (DeYoe \& VanEssen, 1985; Hubel \& Livingstone, 1985; Shipp \& Zeki, 1985).

Certain cells in V1 are known to respond to small disparities (Barlow, Blakemore, \& Pettigrew, 1967) and those in V2 to respond to large retinal disparities (Hubel \& Wiesel, 1970; Ramachandran, Clarke, \& Whitteridge, 1977 ) as well as illusory contours (von der Heydt et al.,
1984). If we make the further assumption that cells in V2 might respond to the disparity of illusory contours, it is not inconceivable that stereo capture results directly from synergistic interactions between cells in these two visual areas. For instance, the repeating texture of the wallpaper might excite cells corresponding to several depth planes in V1, whereas the disparity of illusory contours would excite only a single plane in V2. The latter signal might then be fed back to V1 to select, or "highlight," the appropriate plane-a conjecture that is consistent with the observed anisotropy (Figures $5 \mathrm{a}$ and $5 \mathrm{c}$ ) and phase sensitivity of stereo capture.

Unfortunately, not all aspects of stereo capture can be explained in terms of this simple model. For example, the fact that some degree of capture can be seen even during binocular rivalry is inconsistent with the model (unless one assumes that, in addition to the synergistic process outlined above, there is also a less refined process of "attribution" analogous to motion capture). Also, how does one account for the crucial role played by occlusion in generating such effects as the "porthole" illusion? And finally, how might the activity of nerve cells signal the presence or absence of transparency? We are compelled to admit, reluctantly, that these remarkable percepts may require decision processes higher up along the visual pathways, although it is a comforting thought that at least some aspects of the phenomenon are consistent with the known physiology.

\section{REFERENCES}

Barlow, H. B., Blakemore, C., \& Pettigrew, J. D. (1967). The neural mechanism of binocular depth discrimination. Journal of Physiology, 193, 327-342.

BLOMFIELD, S. (1973). Implicit features and stereoscopy. Nature: New Biology, 245, 147.

BRADDICK, O. J. (1974). A short range process in apparent motion. Vision Research, 14, 519-527.

Brady, M., \& GrImSON, W. E. (1981). The perception of subjective surfaces MIT (AI Memo No. 666). Cambridge, MA: Massachusetts Institute of Technology.

Cavanagh, P., Bogglin, J., \& Favreau, O. (1985). Perception of motion in equiluminous kinematograms. Perception, 14, 151-162.

CoLLETT, T. S. (1985). Extrapolating and interpolating surfaces in depth. Proceedings of the Royal Society, London, B, 224, 43-56.

Coren, S. (1972). Subjective contours and apparent depth. Psychological Review, 79, 359-367.

DeYoe, E. A., \& Van Essen, D. C. (1985). Segregation of efferent connections and receptive field properties in visual area V2 of the Macaque. Nature, 317, 58-61.

GREGORY, R. L. (1972). Cognitive contours. Nature, 238, 51-52.

GrEgoRY, R. L. (1977). Vision with isoluminant colour contrast. Perception, 6, 113-119.

GREGORY, R. L., \& HARRIS, J. (1974). Illusory contours and stereo depth. Perception \& Psychophysics, 15, 411-416.

Helmholtz, H. L. F. voN (1925). Treatise on physiological optics (J. P. Southall, Trans.). New York: Dover. (Original work published 1909)

Hubel, D. H., \& Livingstone, M. S. (1985). Complex unoriented cells in a subregion of primate area 18 . Nature, 315, 325-327.

Hubel, D. H., \& Wiesel, T. N. (1970). Cells sensitive to binocular depth in area 18 of the macaque monkey cortex. Nature, 225, 41-42.

Julesz, B. (1971). Foundations of cyclopean perception. Chicago: University of Chicago Press. 
Julesz, B., \& CHANG, J. J. (1976). Interaction between pools of disparity detectors turned to different disparities. Biological Cybernetics, 22, 107-119.

KanizSA, G. (1976). Subjective contours. Scientific American, 234, 48-52.

KaUFMAN, L. (1964). Suppression and fusion in viewing complex stereograms. American Journal of Psychology, 77, 193-205.

Kaufman, L., \& Pttblado, C. B. (1969). Stereopsis with opposite contrast contours. Perception \& Psychophysics, 6, 10-12.

Lu, C., \& FENDER, D. H. (1972). The interaction of color and luminance in stereoscopic vision. Investigative Ophthalmology, 11, 482-489.

MARR, D. (1982). Vision. San Francisco: Freeman.

Mitchison, G., McKee, S. (1985). Interpolation in stereoscopic matching. Nature, 315, 402-404.

PrazdnY, K. (1985). On the nature of inducing forms generating perceptions of illusory contours. Perception \& Psychophysics, 37, 237-242.

RamaChandran, V. S. (1985a). Apparent motion of subjective surfaces. [In V. S. Ramachandran (Ed.), special issue on motion perception.] Perception, 14, 127-134.

RamaChandRan, V. S. (1985b). Neurobiology of perception: Guest editorial. Perception, 14, 97-103.

Ramachandran, V. S., \& Cavanagh, P. (1985). Subjective contours capture stereopsis. Nature, 317, 527-530.

Ramachandran, V. S., \& CaVANagh, P. (in press). Motion capture anisotropy in random-dot patterns. Vision Research.

Ramachandran, V. S., Clarke, P. G. H., \& Whitteridge, D. (1977). Cells selective to binocular disparity in the cortex of newborn lambs. Nature, 268, 333-335.

RAMACHANDRAN, V. S., \& GREgory, R. L. (1978). Does colour provide an input to human motion perception? Nature, 275, 55-56.

Ramachandran, V. S., \& INADA, V. (1985). Spatial phase and frequency in motion captuure of random-dot patterns. Spatial Vision, 1, 57-67.

Ramachandran, V. S., Rao, V. M., \& Vidyasagar, T. R. (1973). The role of contours in stereopsis. Nature, 242, 412-414.
Rock, I. (1983). The logic of perception. Cambridge, MA: MIT Press. Rogers, B. J., \& GrahaM, M. E. (1979). Motion parallax as an independent cue for depth perception. Perception, 8, 125-134.

SchumanN, F. (1904). Einige Beobachtungen über die Zusammenfassung von Gesichtdrücken zu Einheiten. Psychologische Studien, 1, $1-32$.

SHIPP, S., \& ZEKI, S. M. (1985). Segregation of pathways leading from area V2 to areas V4 and V5 of macaque monkey visual cortex. $\mathrm{Na}$ ture, 315, 322-324.

Treisman, A. (1962). Binocular rivalry and stereoscopic depth perception. Quarterly Journal of Experimental Psychology, 14, 23-27.

UlLMAN, S. (1979). The interpretation of visual motion. Cambridge, MA: MIT Press.

VAN ESSEN, D. C. (1985). Functional organization of primate visual cortex. In A. Peters \& E. G. Jones (Eds.), Cerebral cortex. New York: Plenum.

von der Heydt, R., Peterhans, E., \& Baumgartner, G. (1984). Illusory contours and cortical neuron responses. Science, 224, 1260-1262.

\section{NOTES}

1. A preliminary account of some of these experiments has already been published (Ramachandran \& Cavanagh, 1985).

2. It may turn out that the difference is quantitative rather than qualitative; motion capture may also be anisotropic and phase-sensitive, but to a much smaller extent than stereo capture (Ramachandran \& Cavanagh, in press). If so, one would have to argue that the different weighting functions of the two phenomena reflect differences in natural constraints. A comparative study of capture may therefore provide useful insights into how the brain utilizes these constraints.

(Manuscript received October 21, 1985; revision accepted for publication April 14, 1986.) 